This is the final peer-reviewed accepted manuscript of:

Med Eng Phys. 2020 Oct;84:51-59. doi: 10.1016/j.medengphy.2020.07.024. Epub 2020 Jul 30.

Testing the impact of discoplasty on the biomechanics of the intervertebral disc with simulated degeneration: An in vitro study

Chloé Techens, Marco Palanca, Peter Endre Éltes, Áron Lazáry, Luca Cristofolini

PMID: 32977922 DOI: 10.1016/j.medengphy.2020.07.024

The final published version is available online at:

https://doi.org/10.1016/i.medengphy.2020.07.024

Rights / License:

The terms and conditions for the reuse of this version of the manuscript are specified in the publishing policy. For all terms of use and more information see the publisher's website. 


\title{
Testing the impact of discoplasty on the biomechanics of the intervertebral disc with simulated degeneration: an in vitro porcine study
}

\author{
Chloé Techens, MEng ${ }^{1}$, Marco Palanca, Ph.D. ${ }^{1}$, Peter Endre Eltes MD ${ }^{2}$, \\ Aron Lazary $\mathrm{PhD}^{2}$, Luca Cristofolini, Ph.D. ${ }^{1}$ \\ ${ }^{1}$ Department of Industrial Engineering, School of Engineering and Architecture, Alma \\ Mater Studiorum - Università di Bologna, Bologna, Italy \\ ${ }^{2}$ R\&D Department of National Center for Spinal Disorders, Budapest, Hungary
}

Submitted to: Medical Engineering \& Physics, special issue "Biomechanics for in silico clinical trials: thematic symposium of the IX Meeting of the Italian Chapter of the European Society of Biomechanics"

Original submission: $4^{\text {th }}$ March 2020

Revised version: $\quad 18^{\text {th }}$ July 2020

Statistics:

Word count (manuscript): @ 4890 words

Word count (abstract): @238words

Figures: @9

Tables: @2

References: @ 43

Corresponding author:

Luca Cristofolini

Department of Industrial Engineering

School of Engineering and Architecture

University of Bologna

Viale Risorgimento, 2

40136 Bologna, Italy

e-mail: luca.cristofolini@unibo.it 


\section{ABSTRACT}

2 Percutaneous Cement Discoplasty has recently been developed to relieve pain in highly

3 degenerated intervertebral discs presenting a vacuum phenomenon in patients that

4 cannot undergo major surgery. Little is currently known about the biomechanical effects

5 of discoplasty. This study aimed at investigating the feasibility of modelling empty discs

6 and subsequent discoplasty surgery and measuring their impact over the specimen

7 geometry and mechanical behavior. Ten porcine lumbar spine segments were tested in

8 flexion, extension, and lateral bending under 5.4 Nm (with a $200 \mathrm{~N}$ compressive force

9 and a $27 \mathrm{~mm}$ offset). Tests were performed in three conditions for each specimen: with

10 intact disc, after nucleotomy and after discoplasty. A 3D Digital Image Correlation

11 (DIC) system was used to measure the surface displacements and strains. The posterior

12 disc height, range of motion (ROM), and stiffness were measured at the peak load. CT

13 scans were performed to confirm that the cement distribution was acceptable.

14 Discoplasty recovered the height loss caused by nucleotomy $(\mathrm{p}=0.04)$ with respect to

15 the intact condition, but it did not impact significantly either the ROM or the stiffness.

16 The strains over the disc surface increased after nucleotomy, while discoplasty

17 concentrated the strains on the endplates. In conclusion, this preliminary study has

18 shown that discoplasty recovered the intervertebral posterior height, opening the

19 neuroforamen as clinically observed, but it did not influence the spine mobility or

20 stiffness. This study confirms that this in vitro approach can be used to investigate

21 discoplasty.

\section{Keywords:}

23 Percutaneous Cement Discoplasty, Spine, Biomechanical testing, Strain 


\section{INTRODUCTION}

25 Intervertebral Disc (IVD) degeneration is one of the main causes of low back pain, a

26 large socio-economic burden for society, affecting between $60 \%$ and $70 \%$ of the

27 population in industrialized countries at least once during their lifetimes [1]. Interbody

28 fusion with the insertion of an intervertebral spacer after performing disc fenestration is

29 the most common surgical treatment and has been widely studied in the literature [2]-

30 [10]. It requires an invasive surgery which lasts for hours and is often associated with

31 significant blood loss, long recovery, and general anaesthesia which is not suitable for

32 elderly patients or those with significant comorbidities. Since this disease appears with

33 age, finding minimally invasive treatments is crucial to treat the most complex cases.

34 Percutaneous Cement Discoplasty (PCD), a surgical technique that minimizes the

35 surgical morbidity and complication risks, is applied when a vacuum phenomenon is

36 observed inside the IVD, resulting in the collapse of the adjacent vertebra and in nerve

37 root compression. It consists of injecting an polymethylmethacrylate cement (PMMA)

38 to "create individually shaped "in-site" intervertebral spacers" in order to recover the

39 disc height and decompress the spinal canal [11]. One advantage of using PMMA to

40 stabilize the spine is that "the load-bearing surface of the implant is fully adapted to the

41 shape of the endplates".

43 PCD is a newly developed technique, the authors found very little literature on the 44 subject. Varga et al presented in 2015 the technique and their clinical study on 47 45 patients showed significant improvement in their quality of life, correlating with a pain 46 factor decrease at 6 month follow-up [11]. Another study reported the surgery of a 47 patient treated with PCD [12]. Discoplasty was shown to positively affect the spinal 48 alignment and neuro-foraminal height in 27 patients [13].

49 While the impact of PCD on spine has been clinically assessed by comparing pre50 operative/post-operative scores, no indication about spine kinetics and kinematics has 51 been found by the authors. Some studies investigated similar techniques on animals, 52 performing in vitro testing of spines in intact condition (with a full IVD), after removal 53 of the Nucleus Pulposus (NP), and/or after a stabilization surgery. Refilling of the disc 54 with soft materials [14] to recover intact spine mechanics was also investigated, 
55 however it differs from discoplasty which uses acrylic cement. Only Moissonier et al 56 and Wilke et al mimicked the PCD technique, implanting a spacer within the empty disc.

57 The first demonstrated that nucleotomy of canine IVD increased the Range of Motion 58 (ROM) and reduced disc height, whereas the presence of a hard mass inside the disc 59 recovered the height loss but left ROM as wide as after nucleotomy [3]. The second 60 attested that bone cement stabilized cervical discs, reducing the ROM compared to an 61 intact spine [15]. Moreover, using animal surrogates usually limits access to naturally 62 degenerated discs, consequently research has also focused on the best technique to 63 model the vacuum phenomenon [16], [17], and the mechanical consequences of that 64 surgery [18], [19]. In conclusion, PCD surgery relies on a weak knowledge of the 65 mechanics of lumbar spine treated this way.

66 This study aims at enlarging knowledge about the mechanical consequences of PCD on 67 lumbar spine stability. The motivations were two-fold: first, to develop a method to 68 artificially represent a vacuum disc and the surgical technique applied to in vitro 69 specimens, and to check the efficiency of this method as a model of PCD. Secondly, the 70 study aimed at developing a methodology assessing the biomechanics of the spine before 71 and after discoplasty. In particular, we hypothesized that PCD would recover the 72 posterior disc height, affect the mechanical behaviour of the spine and present a damage 73 risk for the surrounding tissue due to cement presence.

\section{2. MATERIALS AND METHODS}

\subsection{Specimens}

76 Ten functional spinal units were transected between T13 and L6 from porcine (sus scrofa 77 domesticus) thoracolumbar spines. The animals were young and healthy porcine 78 (approximately 9 months old and $100 \mathrm{~kg}$ ) sacrificed for alimentary purposes. The 79 specimens were cleaned using surgical tools: all soft tissues were carefully removed 80 from the segment without damaging the vertebra, the facet joints and the intervertebral 81 disc. In order to keep the natural kinetics of the segment while testing, the anterior, 82 supraspinous and posterior ligaments were left intact. Each segment was aligned based 83 on the disc orientation, using a six-degree-of-freedom clamp. Both segment extremities 84 were potted with acrylic bone cement. Specimens were stored frozen at $-20{ }^{\circ} \mathrm{C}$ between 85 cleaning and testing phases and between the tests which has been proven not to affect 86 significantly the segment biomechanics [20]. 


\subsection{Surgical procedure}

88 The purpose of the study is to develop a method to investigate the impact of PCD on the

89 biomechanical behaviour of the spine by comparing IVD treated by this technique to

90 degenerated and healthy IVDs. Thus, each specimen was tested in the three conditions

91 sequentially:

92 - intact (INT) with a healthy IVD,

93 - after nucleotomy (NUCL) to simulate the instability of degenerated discs,

94 - after discoplasty (DP) (Fig. 1).

\section{2.3. Nucleotomy}

96 Since the porcine specimens were euthanized before reaching skeletal maturation,

97 degenerated disc instability has been manually simulated by reproducing the vacuum

98 inside of the disc. The specimens were thawed at room temperature. A square incision

99 was performed with a scalpel blade in the annulus fibrosus on the latero-posterior side

100 of the disc. The nucleus pulposus, easily identified due to its softness, was completely

101 extracted through the excision with a curette. The endplates were shaved by scratching

102 off the soft tissue until the surfaces felt smooth. This did not weaken the endplates, as

103 no intravertebral leakage was observed during discoplasty. The size of the incision

104 corresponded to the disc height. The specimens were frozen at $-20{ }^{\circ} \mathrm{C}$ until testing.

\section{2.4. Discoplasty}

106 After being tested in degenerated conditions, the specimens were treated with 107 discoplasty. For that, the specimens were thawed at room temperature. A high-viscosity 108 radiopaque acrylic bone cement (10\% BaSO4) (Tecres, Italy) was injected inside the 109 disc through the incision. Because the empty IVD was no longer in tension, the segment 110 was distracted/stretched during the injection to avoid an underestimation of the cement 111 volume. After injection, the cement hardened for $30 \mathrm{~min}$. The specimens were frozen at $112-20{ }^{\circ} \mathrm{C}$ until testing. In one specimen the facet joint was unintentionally damaged at the 113 end of the last test: checking the test results in retrospect confirmed there was no artefact.

\section{2.5. Mechanical testing}

115 All the specimens underwent the same test conditions. In order to simulate in vivo 116 kinetics of porcine spines, a load with offset was applied to simulate flexion, extension, 
117 and lateral bending (the same side was selected for each specimen based on the possible

118 damages made during the preparation). This simplified loading scenario was chosen as

119 it allows reproducible simulation of a realistic loading scenario. In quadrupeds, the

120 choice of a side is less significant than for humans since they do not have a predisposed

121 limb side. The specimens were mechanically tested with a uniaxial servo-hydraulic

122 testing machine (Mod. 8032, Instron, UK) operated in displacement control. The upper

123 pot was rigidly fixed to the top of the testing machine while the other was loaded through

124 a spherical joint moving along a rail (Fig. 1). Before testing, each specimen was thawed

125 at room temperature and pre-conditioned applying a sinusoidal loading at $0.5 \mathrm{~Hz}$ for 20

126 cycles to minimize viscoelastic creep effect. Specimens were loaded at $5.4 \mathrm{Nm}$ by

127 applying $200 \mathrm{~N}$ with an offset of $27 \mathrm{~mm}$. The loading ramp lasted $1 \mathrm{~s}$ then the maximum

128 loading was maintained for $0.3 \mathrm{~s}$ and the specimen was unloaded. The cycle was

129 repeated 6 times (Fig. 2). Three cycles were found to be sufficient for preconditioning

130 the data in another study [21], further cycles being nearly identical. The same trend was

131 observed in these tests. The loading conditions were selected within the range of

132 biological conditions, similar to other past studies [7], [14], [22]-[25]. Besides, the

133 selected load avoided specimen damage. Each test was repeated twice to prove the

134 experiment repeatability. Data extracted from the last cycle of both runs were averaged

135 for each specimen. Axial load and displacement were acquired by the DIC system

136 connected to a load cell $(100 \mathrm{kN})$ at $15 \mathrm{~Hz}$. Additionally, to have a more reliable

137 sequence, the data were recorded with an independent computational unit (PXI,

138 Labview, National Instruments, Aus. Texas, US) at $500 \mathrm{~Hz}$. Unfortunately, some of the

139 former records were missing for the first tests. Loads were either interpolated to have

140 more data or smoothed with a median filter depending of the acquisition frequency.

141 2.6. Displacement and strain with DIC

142 For each test, the specimen surface has been studied using a Digital Image Correlation

143 set-up in order to track its displacements and strains. This technique requires a high-

144 contract speckle pattern on the region of interest. Thus, a white-on-black speckle pattern

145 was prepared on both the vertebra and the intervertebral disc (Fig. 1). First, the segment

146 was stained 3 times with a methylene blue solution to create a dark background without

147 impacting the properties of the tissues [26]. The white pattern was then sprayed

148 following a procedure optimized elsewhere [27]. To measure the displacements and the

149 deformations over the specimen surface, a 3D-DIC system (Q400, Dantec Dynamics, 
150 Skovlunde, Denmark) and the associated software (Instra 4D, v.4.3.1, Dantec

151 Dynamics) were used. Images were acquired by two cameras (5 Megapixels, $2440 \mathrm{x}$

1522050 pixels, 8-bit) with high-quality $35 \mathrm{~mm}$ lenses (Apo-Xenoplan 1.8/35, Schneider-

153 Kreuznach, Bad-Kreuznach, Germany) inclined at an angle of $26^{\circ}$ (white dot line on

154 Fig. 1). The field of view covered the entire specimen (about $50 \mathrm{~mm}$ by $30 \mathrm{~mm}$ ), which

155 gave a pixel size of about $0.02 \mathrm{~mm}$. The specimen was lit by cold-light LEDs. Before the

156 tests, calibration of the DIC system was performed using a dedicated target (A14-BMB-

157 9x9, Dantec Dynamics). The parameters for the images acquisition and the correlation 158 analysis have been previously optimized to minimize the error: facet size of 35 pixels,

159 grid spacing of 11 pixels, and local filtering with a $7 \times 7$ pixels kernel. In order to

160 investigate the biomechanical behaviour of the spine, two different acquisitions were 161 performed:

162 For extension and flexion: Lateral view of the segment with the cameras pointing 163 at the neuroforamen

164 - For lateral bending; Frontal view of the specimen with the cameras pointing to 165 the selected bending side

166 Images were taken at $15 \mathrm{~Hz}$ from the unloaded condition (reference frame, no load 167 applied) to the end of the 6th cycle.

\section{2.7. Data analysis and statistics}

169 The parameters were extracted from the last load cycle of each of the two repetitions of 170 each loading configuration. All measurements were compared for each specimen 171 between the three disc conditions: intact, nucleotomy, discoplasty. In order to assess the 172 changes in the nerve space in the neuroforamen, which is the main point in doing 173 discoplasty, the posterior disc height was measured using DIC images: one point on each

174 endplate was identified on the 3D profile of the disc in the back of the disc, close to the 175 neuroforamen, where the nerve is passing. The points were aligned in the cranial-caudal 176 direction. Their position was therefore tracked using DIC software. As a result, posterior 177 disc height was only computed in flexion and extension, the frontal view not allowing 178 height computation in lateral bending.

179 Displacements of the caudal vertebra in relation to the cranial vertebra were calculated 180 from DIC images with a Matlab script. Assuming vertebra to be rigid bodies, the motions 181 (translations and rotations) of each vertebra were computed based on singular value 
182 decomposition. The ROM was defined as the relative angle between the vertebra in the

183 sagittal plane between the peak load and unloaded conditions.

184 A pilot study of the load-displacement curves determined that, for porcine spines, the 185 position having a first derivative of $30 \mathrm{~N} / \mathrm{mm}$ was at the limit of the laxity zone (LZ).

186 Stiffness was defined as the slope of load-displacement relationship in LZ. Although for 187 some specimens this method underestimated the length of the LZ, the stiffness 188 computation was not impacted since it was within the linear region [28].

189 All the computations were performed with dedicated Matlab scripts (Mathworks, Inc.,

190 Natick, MA, USA). All height and strain measurements were evaluated by two 191 independent observers. To limit inter-specimen variability influence, all stiffnesses, 192 heights, and ROMs values were normalized to the intact condition.

193 In addition to posterior disc height, ROM, and stiffness calculations, the true principal 194 strains over the specimen surface (vertebra and IVD) were measured at the peak load. 195 In particular, the disc surface area was manually identified and the minimum, maximum 196 and average of the first and second principal strains were extracted. Those measurements 197 were performed in flexion and extension because the frontal view did not allow 198 consideration of the neuroforamen area.

199 For each parameter, outlying data were preliminarily tested and excluded using the 200 Peirce criterion [29], this resulted in a $10 \%$ data exclusion at the maximum. Test 201 parameters were computed based on the sixth cycle. Mean \pm standard deviation of all 202 the outcomes were calculated and presented by group. Due to the small specimen 203 number, comparisons between the three conditions were made for ROM, stiffness, 204 height, and the strain average with a non-parametric test (Wilcoxon's sign rank, with 205 Matlab).

206 2.8. Cement distribution

207 In order to study the cement distribution inside of the disc, scans of the specimens have 208 been performed after discoplasty with a clinical computed tomography scanner 209 (Aquilion ONE, Toshiba) with $120 \mathrm{~mA}, 135 \mathrm{kV}$ and a $0.5 \mathrm{~mm}$ voxel. The scans of nine 210 specimens out of ten were available due to a practical mistake. The shape of the cement, 211 its capacity to fill the disc cavity, the endplates and AF contact were visually assessed 212 by a spine surgeon (P.E.) from the 3D reconstruction of the PMMA geometry. 213 Segmentation process was performed in Mimics ${ }^{\circledR}$ image analysis software (Mimics 
214 Research, Mimics Innovation Suite v21.0, Materialise, Leuven, Belgium) on the 2D CT

215 images using thresholding algorithm.

\section{3. RESULTS}

\section{3.1. Posterior disc height}

218 The posterior disc height was measured in the three conditions. At peak load, intact

219 posterior disc height was higher in flexion than in extension. Nucleotomy significantly

220 decreased the posterior height for both flexion $(\mathrm{p}=0.006$, Wilcoxon) and extension

221 ( $\mathrm{p}=0.049$, Wilcoxon) (Fig. 3). On the contrary, discoplasty restored the height. Results

222 were normalized to the initial posterior height for each specimen. In extension, height

223 after discoplasty was significantly higher (105\% of the intact height) than after

224 nucleotomy $(81 \%)(\mathrm{p}=0.04$, Wilcoxon). In flexion, posterior disc height was

225 respectively $84 \%$ and $94 \%$ of the intact height after nucleotomy and discoplasty but the

226 difference between the two conditions was not significant ( $\mathrm{p}=0.11$, Wilcoxon).

\section{3.2. Range of motion}

228 Intervertebral motions in the applied direction were one order of magnitude higher

229 compared to the other directions. Only the motions in the applied direction are reported

230 here. In flexion and lateral bending, nucleotomy reduced the ROM (Fig. 4). The ROM

231 in extension slightly increased after nucleotomy and discoplasty compared to the intact

232 condition. The results for degenerated and discoplasty discs were normalized by the

233 intact ROM for each motion. ROM was not significantly different between nucleotomy

234 and discoplasty in flexion (Wilcoxon sign-rank test, $p=0.57)$, extension $(p=0.43)$ and

235 lateral bending ( $\mathrm{p}=0.50$, Wilcoxon).

\section{3.3. Stiffness}

237 Stiffness was computed for only 9 out of 10 specimens due to a technical problem during

238 acquisition. Specimens had very different behaviours regardless of the loading

239 configuration and spinal level. The majority of the tests presented a "toe-region" before

240 a stiff region. A recovery after discoplasty of the initial behaviour compared to after

241 nucleotomy was also observed (Fig. 5). The results for nucleotomy and discoplasty discs

242 were normalized by the intact stiffness for each loading configuration. Stiffness was not 
243 significantly different after nucleotomy and discoplasty in flexion ( $\mathrm{p}=0.47$, Wilcoxon),

244 extension ( $p=0.95$, Wilcoxon) and lateral bending ( $p=0.46$, Wilcoxon) (Fig. 6).

\section{3.4. Strain distribution}

246 DIC correlation has been successfully performed in flexion and extension only because

247 the frontal view did not allow all of the disc surface to be captured. First of all, bone 248 strains were in a $[-1.5 \%, 1.5 \%]$ range on the vertebra surface whereas they reached $-17 \%$ 249 and $+11 \%$ on the discs. Moreover, IVD principal strains presented different behaviours 250 depending on the loading configuration (Fig. 7). In flexion, for all disc conditions, the 251 highest values of compressive strain are located at the cranial and caudal extremities of 252 the IVD, starting from the anterior and spreading toward the posterior along the 253 endplates. After nucleotomy and discoplasty, the trend was more pronounced. However, 254 cemented discs presented lower values in this area than empty discs. The highest values 255 of tensile principal strain were in the centre of the IVD with peak $>3 \%$ of strain in the 256 posterior region. In extension, tensile strains were larger in the anterior of the disc while 257 high compressive strains were located in the posterior area of the disc. Discoplasty 258 reduced the strains in most of the disc, whereas for intact and nucleotomy, high strains 259 were found on the whole disc.

260 Nucleotomy seems to have a greater effect on the compressive strain in flexion and 261 extension (Table 1). Meanwhile, discoplasty halved the average tensile strain of disc 262 surface compared to nucleotomy condition in extension ( $\mathrm{p}=0.0195$, Wilcoxon) but had 263 similar values of second principal strain. Regarding the peak strains, discoplasty only 264 presented a value larger than intact condition for extension. Other extreme strains were 265 observed after nucleotomy although the differences were not significant.

\section{3.5. Cement distribution}

267 Nine specimens have been scanned to control cement distribution within the discs.

268 Visual assessment of the specimen scans focused on the position of the cement mass 269 within the intervertebral disc in the sagittal and frontal planes, whether it was in contact 270 with endplates and AF, the shape of the distribution, and the ratio of disc filling. The 271 majority of specimens had a cement volume located in the posterior of the disc (9/9 272 specimens), centred in the lateral direction ( $8 / 9$ specimens), in contact with the endplates 
273 (8/9). Only two specimens did not present contact between the cement and the AF (Fig.

274 8). The NP cavity was fully filled with cement in 5 specimens, three discs were almost 275 filled at $>80 \%$ of the NP volume, and one at less than $80 \%$. Among the specimens, seven 276 were validated by a clinician as discoplasty models compared to cement distribution 277 after human surgery taking porcine anatomical specificities into account, and two were 278 sub-optimal (Fig. 9). No outlier corresponded to the sub-optimal cemented specimens.

279 All specimens presented a smaller cement volume than in human surgery (Table 2).

\section{4. DISCUSSION}

281 According to clinical observations [11], a loss of disc height due to disc degeneration 282 would result in a reduction of the neuroforamen where the back nerves are passing, 283 compressing them and creating pain for the patient. This animal in vitro study aimed at 284 exploring the feasibility of assessing the mechanical consequences on spine stability 285 after discoplasty surgical procedure. An in vitro experiment was successfully conducted 286 to establish posterior disc height, ROM, stiffness, and strains over porcine specimen 287 surfaces.

288 After nucleotomy a decrease of the posterior disc height of $15 \%$ was measured. This 289 result validated such in vitro nucleotomy as a simulation of degenerated disc. 290 Furthermore, nucleotomy was associated with a decrease of ROM (not statistically 291 significant in our sample). After discoplasty, the injected cement acted like a spacer 292 resulting in a significant recovery of the posterior height (105\% of the intact height in 293 extension). This trend supported the clinical observations [11] and confirmed that PCD 294 recovered the disc height and enlarged neuroforamen space, which is the main objective 295 of this surgery. ROM and stiffness did not show any significant difference between the 296 degenerated and treated cases for any loading. Thus, discoplasty did not significantly 297 impact spine flexibility in this experimental setup.

298 To the authors' knowledge, this was the first study addressing the consequences of 299 discoplasty on the distribution of strain on the disc surface. The strain distribution 300 measured after nucleotomy showed a specific pattern with intense regions, while 301 discoplasty reduced this abnormal distribution with more moderate strain values.

302 DIC results showing the AF principal strains can be related to the ROM and the posterior 303 disc height. After nucleotomy, because of the reduced posterior height and because the 304 annulus is no longer constrained from inside, the annulus fibres bulged more, leading to 
305 intense tensile strains at the apex of the bulging. At the same time, this more pronounced

306 bulging at mid-height caused a more pronounced concavity at the disc cranial and caudal

307 extremities, which led to larger compressive strains in this region. After discoplasty, the

308 injected cement spaced the endplates, and even if the cement did not stretch radially the

309 disc fibres as the NP would do, the overall bulging was more limited, and less intense

310 tensile strains were measured. As the cement acts as a very stiff spacer, very small strains

311 were visible in most of the disc surface, the only highly strained region in the disc was

312 near the endplates. Strain values after discoplasty did not exceed what the endplates

313 underwent in nucleotomy condition. If the specimen endplates presented any weakness,

314 this could lead to long-term damages due to the load concentration. The peak strain

315 values increased after nucleotomy, and decreased again after cement injection, reaching

316 intact-like values. No correlation between the strain peaks on the specimen surface and

317 the cement distribution assessed from the CT scans was found. Even in the specimens

318 where contact between the AF and the cement was noted, this did not result in a specific

319 strain distribution.

320 The ROM measured at peak load was in the same range as other in vitro studies on 321 porcine lumbar spines [22], [30]. Others studies investigating the effect of nucleotomy 322 demonstrated that the absence of NP reduced segmental rotational stability, significantly 323 increasing the ROM [14], [19], [23].

324 Discoplasty being a recent surgical technique, the authors found only one article 325 applying a similar surgery, on dog cervical discs [3]: nucleotomy was also performed 326 through an AF fenestration and a spacer implant was inserted. Similar to the present 327 study, Moissonier et al found that nucleotomy completely disrupted spine stability, 328 increasing significantly the ROM. Both the spacer used in their study, and the cement 329 injected in ours failed to recover disc mobility. Similarly, the cement set in the cervical 330 disc by Wilke et al reduced the ROM compared to intact disc condition. However, this 331 study tested bone cement to anticipate interbody fusion, and the AF was not fully intact 332 [15]. This was the major difference with soft disc filler materials which are more likely 333 to restore intact ROM as well [14].

334 Although the results were normalized with respect to the intact to integrate the specimen 335 anatomical specificity, and one outlier was removed, inter-specimen variability 336 remained large, with no correlation with the segment level. Our tests differed from most 337 of the literature [28] as the FSUs were tested separately in flexion and extension, 338 therefore direct comparisons of the stiffness are not possible. 
339 This study aimed to start exploring the biomechanical effects of discoplasty. Since this

340 is a preliminary study, an animal model was more justifiable for ethical reasons. The

341 use of breed porcine rather than human spines was preferred as they have less inter-

342 specimen variation of anatomy and mechanical properties. Indeed, porcine models are

343 commonly used to replicate human spine surgeries [31], [32]. Porcine spines could be

344 good surrogates for in vitro testing, even if they exhibit larger ROM and lower stiffness

345 [33]-[35]. Since the porcine specimens were obtained before reaching skeletal maturity,

346 finding IVDs presenting a similar degenerated level with a vacuum as required for PCD

347 was impossible. Nucleotomy did not aim at modelling a degenerated disc state but at

348 creating the spine instability observed clinically based on the disc vacuum. Porcine

349 results should therefore be qualitatively extrapolated to humans in terms of trends rather

350 than interpreting absolute values as this study aimed at.

351 Vacuum volume has not been measured in this study. The importance of this parameter

352 is unclear in the clinical practice. A recent study investigating the Vacuum Phenomenon

353 (VP) impact for PCD indication concluded that the volume of vacuum could not be used

354 as a proper indication for this surgery [36]. Moreover, during the PCD procedure, the

355 patient position aims at enlarging the intervertebral space by reducing the segmental

356 lordosis. Thus, the volume of the empty disc available during the surgery is larger than

357 the VP computed on imaging.

358 Usual methods to measure the disc height like Farfan or Frobin were not applied here to 359 assess the intervertebral space. Indeed, these methods were conceived for clinical 360 application considering the vertical height along the antero-posterior disc length on 361 medical images, taking account of the whole disc and its orientation. This study, 362 however, focused on the nerve space within the neuroforamen. Only the volume where 363 the nerve passed was critical, based on clinical observations, and the discoplasty surgery 364 was applied in first approach to re-open the foramen space by achieving indirect 365 decompression. That is why a comparative study has been performed selecting two 366 points at the endplates level the closest from the neuroforamen, rather than relying on a 367 more general measurement of the disc height. The study concentrated on parameters 368 with meaning for the clinical purpose of the surgery. Moreover, the most critical case 369 was also investigated: physiologically when the disc is loaded in extension and the 370 neuroforamen is the most reduced. So, measurements at the peak load were more 371 interesting for the study. 
372 The impact of AF fenestration during nucleotomy on the segment stability has not been

373 assessed here, however Michalek et al reported alterations of IVD mechanics with disc

374 height loss under a compressive load, following different types of incisions [37]. Disc

375 lesions were also found to reduce the peak moment depending on the damage shape [38].

376 As a consequence, our study may overestimate motion range. However, it was

377 hypothesized that the lack of NP would destabilize the segment in larger proportion than

378 the fenestration of AF.

379 Pure moment is the gold-standard loading for in vitro spine testing in a relevant bending 380 condition. For spine segments consisting of several vertebrae, bending is usually 381 associated with a follower load equipment to model the in vivo kinematics, including the 382 effect of the muscles adding a compressive loading [39], [40]. Similarly, a compressive 383 preload is found in a single FSU, but in this case a follower load cannot be implemented.

384 In this study, an alternative loading configuration was chosen to ensure that reproducible

385 testing conditions could be applied, thus allowing the comparison of the biomechanics 386 of a specimen tested at different times with each of the different disc conditions. The 387 load applied here was a combination of axial compression and bending, an alternative 388 loading to pure bending of the spine [26], [41]-[44]. It has been demonstrated that 389 without preload, in vivo stiffness of the spine segment was underestimated applying pure 390 bending [45]. In our study, the combination of axial compression and bending allowed 391 a more physiological spine loading with an axial component which substitutes of the 392 preload.

\section{5. CONCLUSION}

394 So far, the only knowledge about PCD comes from clinical experience on few cases. 395 This paper presents a feasibility study, to develop a test model and perform a preliminary 396 investigation on the biomechanics of PCD. The study also aimed at analyzing and 397 verifying if there is any clear mechanical risk associated with injection of cement in the 398 cavity of a disc. No specific clinical recommendations (e.g. indication for specific 399 patient groups) can be directly obtained from the present study. This study aimed at 400 developing an in vitro surrogate to test a highly degenerated disc with vacuum inside, 401 and to assess the biomechanical changes related to discoplasty in porcine spines. The 402 main conclusions could be summarized in key points. 
- The in vitro method was successfully developed to model nucleotomy.

- The in vitro testing protocol applied to discoplasty allowed to measure the effect of this minimally invasive surgery on the spine biomechanics.

- Nucleotomy decreased the posterior disc height. Discoplasty restored the height significantly, maintaining a gap between the vertebral bodies and re-opening the neuroforamen area as observed in clinical practice.

- Discoplasty did not impact the ROM nor the stiffness, which remained similar

- The CT scans confirmed that the distribution of the cement had a similar distribution inside the disc for most specimens compared to human post-surgery observations, although the cavity after nucleotomy and the cement volume were smaller than in human cases.

- The goal of this preliminary study on a limited number of porcine specimens was to establish trends which could justify a larger study on human specimens.

\section{Acknowledgments}

422 The Authors wish to thank Federico Morosato from the University of Bologna for 423 providing the Matlab scripts.

424 Villalba Hospital is acknowledged for hosting the scan sessions; special thanks to 425 Pierangela Moro for the skilled advice and for her great patience.

426 Special thanks are expressed to Cameron James, ESR within the Spinner project, for 427 proof-reading the manuscript.

428 The use Mimics Software was possible thanks to the Hungarian Scientific Research 429 Fund (OTKA FK123884).

430 This project was founded by European Union's Horizon 2020 Marie Skłodowska-Curie 431 ITN grant SPINNER No. 766012. 


\section{Conflict of interest statement}

433 There is no potential conflict of interest: none of the Authors received or will receive

434 direct or indirect benefits from third parties for the performance of this study.

435 
[1] «6. Priority diseases and reasons for inclusion », in WHO $\mid$ Priority Medicines for Europe and the World Update Report, 2013.

[2] I. Oda, K. Abumi, B.-S. Yu, H. Sudo, et A. Minami, « Types of Spinal Instability That Require Interbody Support in Posterior Lumbar Reconstruction: An In Vitro Biomechanical Investigation. [Miscellaneous Article] », Spine, vol. 28, n 14, p. 1573-1580, juill. 2003.

[3] P. Moissonnier, L. Desquilbet, D. Fitzpatrick, et F. Bernard, « Radiography and biomechanics of sixth and seventh cervical vertebrae segments after disc fenestration and after insertion of an intervertebral body spacer », Vet. Comp. Orthop. Traumatol., vol. 27, no 1, p. 54-61, 2014, doi: 10.3415/VCOT-11-110159.

[4] X. Li, Y. Song, et H. Duan, « Reconstruction of Segmental Stability of Goat Cervical Spine with Poly (D, L-lactic acid) Cage », Orthop. Surg., vol. 7, no 3, p. 266-272, 2015, doi: 10.1111/os. 12192.

[5] F. Kandziora, R. Pflugmacher, M. Scholz, T. D. Eindorf, K. J. Schnake, et N. P. Haas, « Bioabsorbable Interbody Cages in a Sheep Cervical Spine Fusion Model. », Spine, vol. 29, nº 17, p. 1845-1855, sept. 2004.

[6] F. Kandziora et al., « Comparison of BMP-2 and combined IGF-I/TGF-ß1 application in a sheep cervical spine fusion model », Eur. Spine J., vol. 11, n ${ }^{0} 5$, p. 482-493, oct. 2002, doi: 10.1007/s00586-001-0384-4.

[7] Y. Gu, Z. Yao, L. Jia, J. Qi, et J. Wang, « In vivo experimental study of hat type cervical intervertebral fusion cage (HCIFC) », Int. Orthop., vol. 34, $\mathrm{n}^{\circ} 8$, p. 1251-1259, déc. 2010, doi: 10.1007/s00264-010-0978-8.

[8] Z. Chunguang et al., « Evaluation of Bioabsorbable Multiamino Acid Copolymer/ $\alpha$-Tri-Calcium Phosphate Interbody Fusion Cages in a Goat Model »:, Spine, vol. 36, $\mathrm{n}^{\circ}$ 25, p. E1615-E1622, déc. 2011, doi: 10.1097/BRS.0b013e318210ca32.

[9] M. J. Allen, Y. Hai, N. R. Ordway, C.-K. Park, B. Bai, et H. A. Yuan, " Assessment of a synthetic anterior cervical ligament in a spinal fusion model in sheep », Spine J., vol. 2, nº 4, p. 261-266, juill. 2002, doi: 10.1016/S15299430(02)00188-2.

[10] S. E. Emery, D. A. Fuller, et S. D. Stevenson, « Ceramic Anterior Spinal Fusion: Biologic and Biomechanical Comparison in a Canine Model. [Miscellaneous Article] », Spine, vol. 21, n 23, p. 2713-2719, déc. 1996.

[11] P. P. Varga, G. Jakab, I. B. Bors, A. Lazary, et Z. Szövérfi, « Experiences with PMMA cement as a stand-alone intervertebral spacer », Orthop., vol. 44, $\mathrm{n}^{\circ} 1, \mathrm{p}$. 1-8, nov. 2015, doi: 10.1007/s00132-014-3060-1.

[12] C. Sola et al., « Percutaneous cement discoplasty for the treatment of advanced degenerative disk disease in elderly patients », Eur. Spine J., mars 2018, doi: 10.1007/s00586-018-5547-7.

[13] L. Kiss, P. P. Varga, Z. Szoverfi, G. Jakab, P. E. Eltes, et A. Lazary, « Indirect foraminal decompression and improvement in the lumbar alignment after percutaneous cement discoplasty », Eur. Spine J., avr. 2019, doi: 10.1007/s00586-019-05966-7.

[14] H.-J. Wilke, F. Heuer, C. Neidlinger-Wilke, et L. Claes, « Is a collagen scaffold for a tissue engineered nucleus replacement capable of restoring disc height and stability in an animal model? », Eur. Spine J., vol. 15, n 3, p. 433-438, août 2006, doi: 10.1007/s00586-006-0177-x. 
[15] H.-J. Wilke, A. Kettler, et L. Claes, « Primary stabilizing effect of interbody fusion devices for the cervical spine: an in vitro comparison between three different cage types and bone cement », Eur. Spine J., vol. 9, nº 5, p. 410-416, oct. 2000, doi: 10.1007/s005860000168.

[16] G. Vadalà et al., « A Nucleotomy Model with Intact Annulus Fibrosus to Test Intervertebral Disc Regeneration Strategies », Tissue Eng. Part C Methods, vol. 21, no 11, p. 1117-1124, 2015, doi: http://dx.doi.org/10.1089/ten.tec.2015.0086.

[17] G. Vadalà et al., " The Transpedicular Approach As an Alternative Route for Intervertebral Disc Regeneration »:, Spine, vol. 38, nº 6, p. E319-E324, mars 2013, doi: 10.1097/BRS.0b013e318285bc4a.

[18] M. Shea, T. Y. Takeuchi, R. H. Wittenberg, A. A. White, et W. C. Hayes, « A Comparison of the Effects of Automated Percutaneous Diskectomy and Conventional Diskectomy on Intradiscal Pressure, Disk Geometry, and Stiffness »:, J. Spinal Disord., vol. 7, n 4, p. 317???325, août 1994, doi: 10.1097/00002517-199408000-00005.

[19] W. Johannessen, J. M. Cloyd, G. D. O’Connell, E. J. Vresilovic, et D. M. Elliott, « Trans-Endplate Nucleotomy Increases Deformation and Creep Response in Axial Loading », Ann. Biomed. Eng., vol. 34, n 4, p. 687-696, avr. 2006, doi: 10.1007/s10439-005-9070-8.

[20] J. S. Tan et S. Uppuganti, « Cumulative Multiple Freeze-Thaw Cycles and Testing Does Not Affect Subsequent Within-Day Variation in Intervertebral Flexibility of Human Cadaveric Lumbosacral Spine », SPINE, vol. 37, n 20, p. E1238-E1242, 2012.

[21] J. M. Cottrell, M. C. H. van der Meulen, J. M. Lane, et E. R. Myers, « Assessing the Stiffness of Spinal Fusion in Animal Models », HSS J., vol. 2, n 1, p. 12-18, févr. 2006, doi: 10.1007/s11420-005-5123-7.

[22] J. P. Dickey et D. J. Kerr, « Effect of specimen length: are the mechanics of individual motion segments comparable in functional spinal units and multisegment specimens? », Med. Eng. Phys., vol. 25, n 3, p. 221-227, avr. 2003, doi: 10.1016/S1350-4533(02)00152-2.

[23] F. Russo et al., « Biomechanical Evaluation of Transpedicular Nucleotomy With Intact Annulus Fibrosus »:, SPINE, vol. 42, no 4, p. E193-E201, févr. 2017, doi: 10.1097/BRS.0000000000001762.

[24] D. J. Sucato, «Thoracoscopic Discectomy and Fusion in an Animal Model: Safe and Effective When Segmental Blood Vessels Are Spared. », SPINE, vol. 27, $\mathrm{n}^{\circ}$ 8, p. 880-886, 2002.

[25] Chung et Teoh, « Multi-axial Spine Biomechanical Testing System with Speckle Displacement Instrumentation », J. Biomech. Eng., vol. 124, nº 4, p. 471-477, août 2002, doi: 10.1115/1.1493803.

[26] M. Palanca, M. Marco, M. L. Ruspi, et L. Cristofolini, « Full-field strain distribution in multi-vertebra spine segments: An in vitro application of digital image correlation », Med. Eng. Phys., vol. 52, p. 76-83, févr. 2018, doi: 10.1016/j.medengphy.2017.11.003.

[27] M. Palanca, T. M. Brugo, et L. Cristofolini, « USE OF DIGITAL IMAGE CORRELATION TO INVESTIGATE THE BIOMECHANICS OF THE VERTEBRA », J. Mech. Med. Biol., vol. 15, nº 02, p. 1540004, avr. 2015, doi: 10.1142/S0219519415400047.

[28] H.-J. Wilke, K. Wenger, et L. Claes, « Testing criteria for spinal implants: recommendations for the standardization of in vitro stability testing of spinal 

implants », Eur. Spine J., vol. 7, n 2, p. 148-154, mai 1998, doi: $10.1007 / \mathrm{s} 005860050045$.

[29] S. M. Ross, « Peirce's criterion for the elimination of suspect experimental data », J. Eng. Technol., p. 1-12, 2003.

[30] J. T. Lysack, J. P. Dickey, G. A. Dumas, et D. Yen, « A continuous pure moment loading apparatus for biomechanical testing of multi-segment spine specimens », J. Biomech., vol. 33, nº 6, p. 765-770, juin 2000, doi: 10.1016/S00219290(00)00021-X.

[31] Busscher, " Comparative anatomical dimensions of the complete human and porcine spine », 2010.

[32] C. Daly, P. Ghosh, G. Jenkin, D. Oehme, et T. Goldschlager, « A Review of Animal Models of Intervertebral Disc Degeneration: Pathophysiology, Regeneration, and Translation to the Clinic », BioMed Res. Int., vol. 2016, 2016, doi: 10.1155/2016/5952165.

[33] H.-J. Wilke, J. Geppert, et A. Kienle, « Biomechanical in vitro evaluation of the complete porcine spine in comparison with data of the human spine », Eur. Spine $J$, vol. 20, no 11, p. 1859-1868, nov. 2011, doi: 10.1007/s00586-011-1822-6.

[34] J. P. Dickey, G. A. Dumas, et D. A. Bednar, « Comparison of porcine and human lumbar spine flexion mechanics* », Vet. Comp. Orthop. Traumatol., vol. 16, $\mathrm{n}^{\mathrm{o}}$ 01, p. 44-49, 2003, doi: 10.1055/s-0038-1632753.

[35] I. Busscher, A. J. van der Veen, J. H. van Dieen, I. Kingma, G. J. Verkerke, et A. G. Veldhuizen, «In Vitro Biomechanical Characteristics of the Spine A Comparison Between Human and Porcine Spinal Segments », SPINE, vol. 35, ${ }^{\circ}$ 2, p. E35-E42, janv. 2010, doi: 10.1097/BRS.0b013e3181b21885.

[36] G. Camino Willhuber et al., « Development of a New Therapy-Oriented Classification of Intervertebral Vacuum Phenomenon With Evaluation of Intraand Interobserver Reliabilities », Glob. Spine J., p. 2192568220913006 , mars 2020, doi: 10.1177/2192568220913006.

[37] A. J. Michalek et J. C. Iatridis, « Height and torsional stiffness are most sensitive to annular injury in large animal intervertebral discs », Spine J., vol. 12, no 5, p. 425-432, mai 2012, doi: 10.1016/j.spinee.2012.04.001.

[38] R. E. Thompson, M. J. Pearcy, et T. M. Barker, « The mechanical effects of intervertebral disc lesions », Clin. Biomech., vol. 19, n 5, p. 448-455, juin 2004, doi: 10.1016/j.clinbiomech.2004.01.012.

[39] A. G. Patwardhan, R. M. Havey, K. P. Meade, B. Lee, et B. Dunlap, « A Follower Load Increases the Load-Carrying Capacity of the Lumbar Spine in Compression. », SPINE, vol. 24, n 10, p. 1003-1009, 1999.

[40] A. G. Patwardhan, K. P. Meade, et B. Lee, « A Frontal Plane Model of the Lumbar Spine Subjected to a Follower Load: Implications for the Role of Muscles », J. Biomech. Eng., vol. 123, n 3, p. 212-217, juin 2001, doi: 10.1115/1.1372699.

[41] M. A. Adams, S. May, B. J. C. Freeman, H. P. Morrison, et P. Dolan, « Effects of Backward Bending on Lumbar Intervertebral Discs: Relevance to Physical Therapy Treatments for Low Back Pain. », SPINE, vol. 25, nº 4, p. 431-437, 2000.

[42] M. Al-Rawahi, J. Luo, P. Pollintine, P. Dolan, et M. A. Adams, « Mechanical Function of Vertebral Body Osteophytes, as Revealed by Experiments on Cadaveric Spines »:, Spine, vol. 36, n 10, p. 770-777, mai 2011, doi: 10.1097/BRS.0b013e3181df1a70. 
[43] M. L. Ruspi, M. Palanca, C. Faldini, et L. Cristofolini, « Full-field in vitro investigation of hard and soft tissue strain in the spine by means of Digital Image Correlation ", Muscles Ligaments Tendons J., vol. 7, no 4, p. 538-545, avr. 2018, doi: $10.11138 / \mathrm{mltj} / 2017.7 .4 .538$.

[44] M. Adams et P. Dolan, « Time-dependent changes in the lumbar spine's resistancc to bending », Clin. Biomech., vol. 11, n 4, p. 194-200, juin 1996, doi: 10.1016/0268-0033(96)00002-2.

[45] M. G. Gardner-Morse et I. A. Stokes, « Physiological axial compressive preloads increase motion segment stiffness, linearity and hysteresis in all six degrees of freedom for small displacements about the neutral posture », J. Orthop. Res., vol. 21, no 3, p. 547-552, janv. 2003, doi: 10.1016/S0736-0266(02)00199-7. 
CAPTIONS TO FIGURES
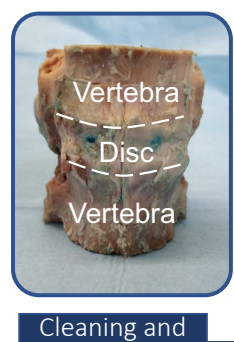

Alignment

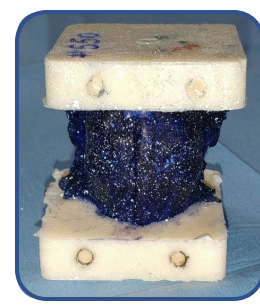

Speckle Pattern

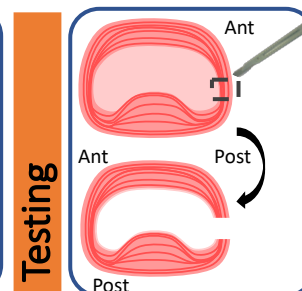

Nucleotomy

.

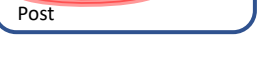

Discoplasty

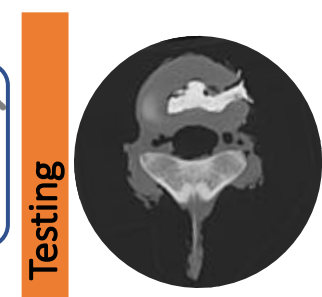

Imaging - CT

598 Fig. 1 - Experimental workflow of the study. The arrow represents the applied load 599 and the resulting moment $\mathrm{M}$. 
600

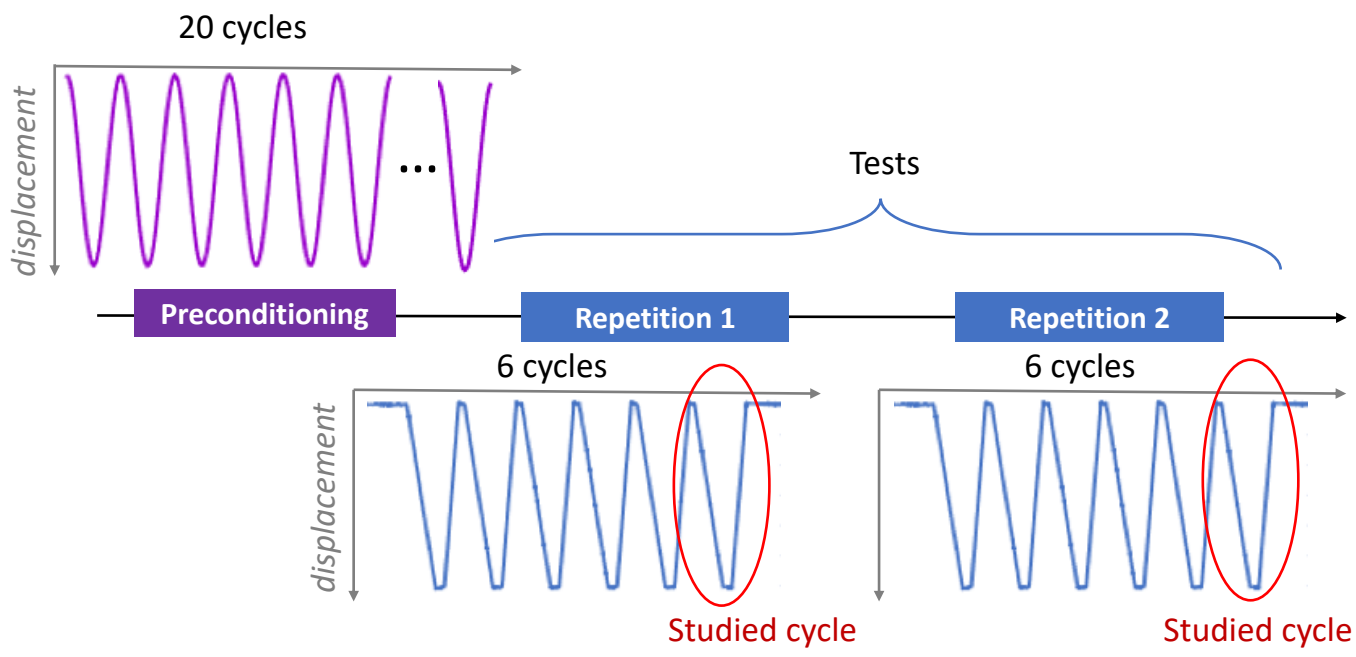

601

602 Fig. 2 - Workflow of the applied displacement for flexion, extension, and lateral 603 bending. 


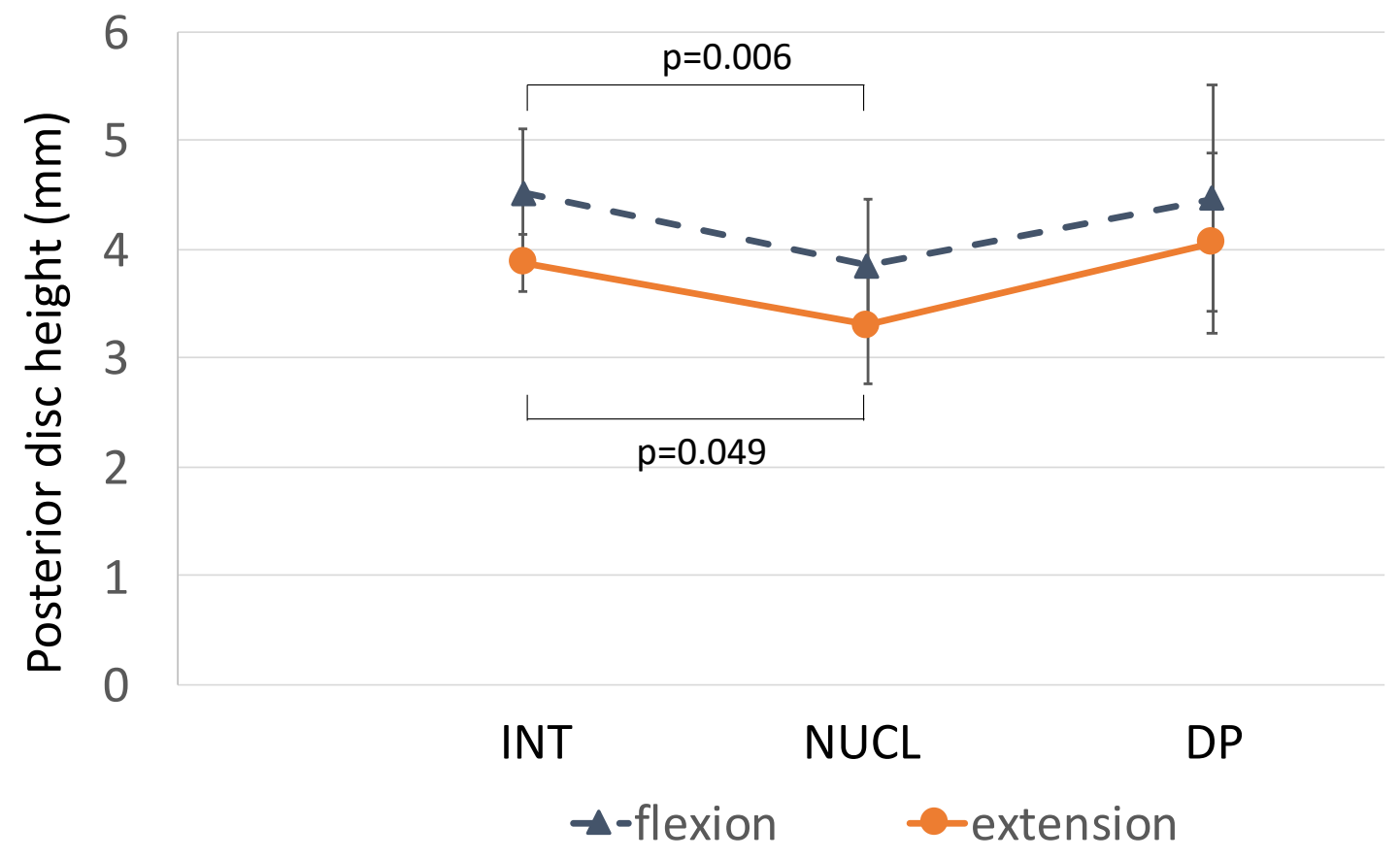

606 Fig. 3 - Intervertebral posterior disc height recorded at the peak load in intact 607 condition, after nucleotomy, and discoplasty for both motions. Average over all 608 specimens and standard deviation were represented $(n=10)$. Normalized data showed 609 significant differences in flexion $(\mathrm{p}=0.11)$ and extension $(\mathrm{p}=0.04)$ between NUCL and 610 DP 


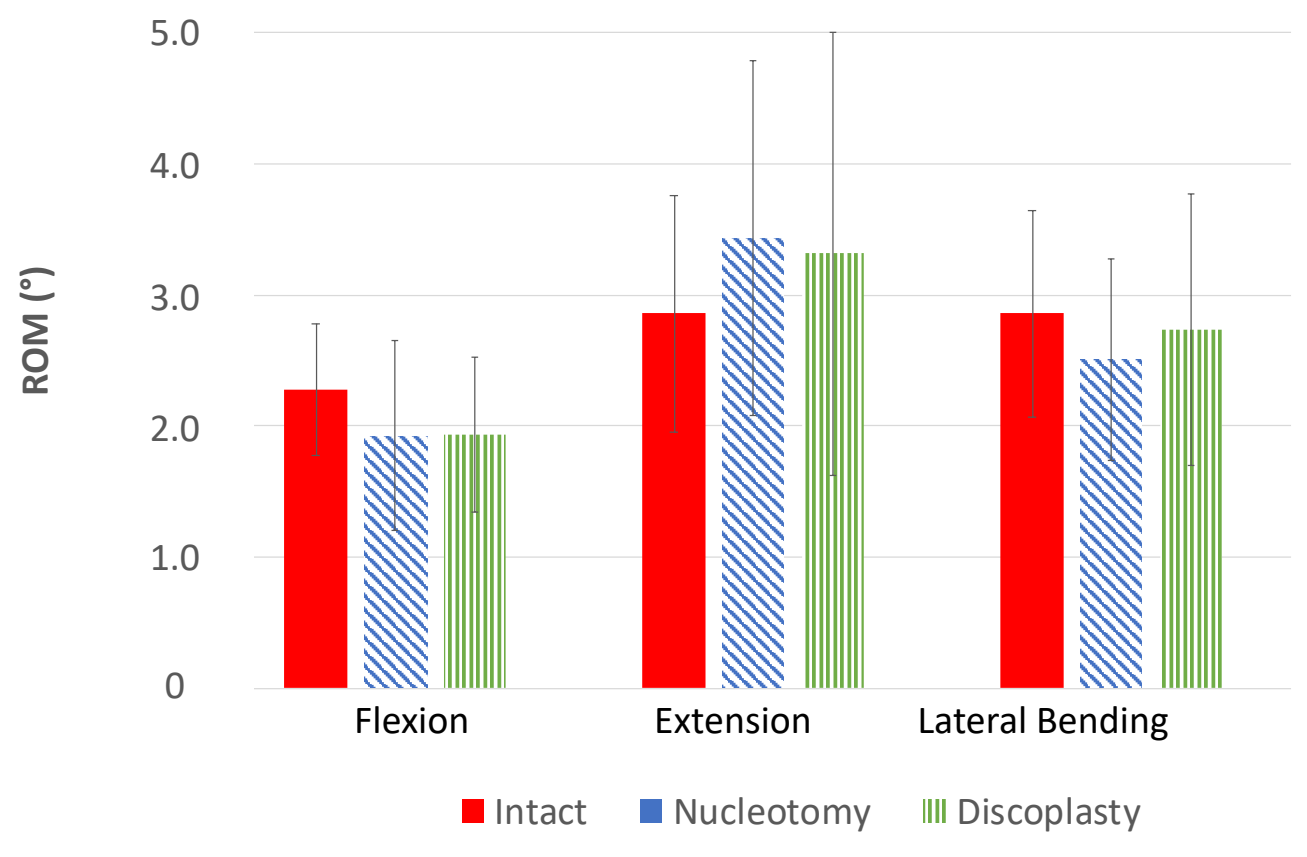

613 Fig. 4 - Range of Motion recorded at peak load for flexion, extension and lateral 614 bending, in all disc conditions. Normalized data were not statistically significant $615(\mathrm{p}>0.1)$. 


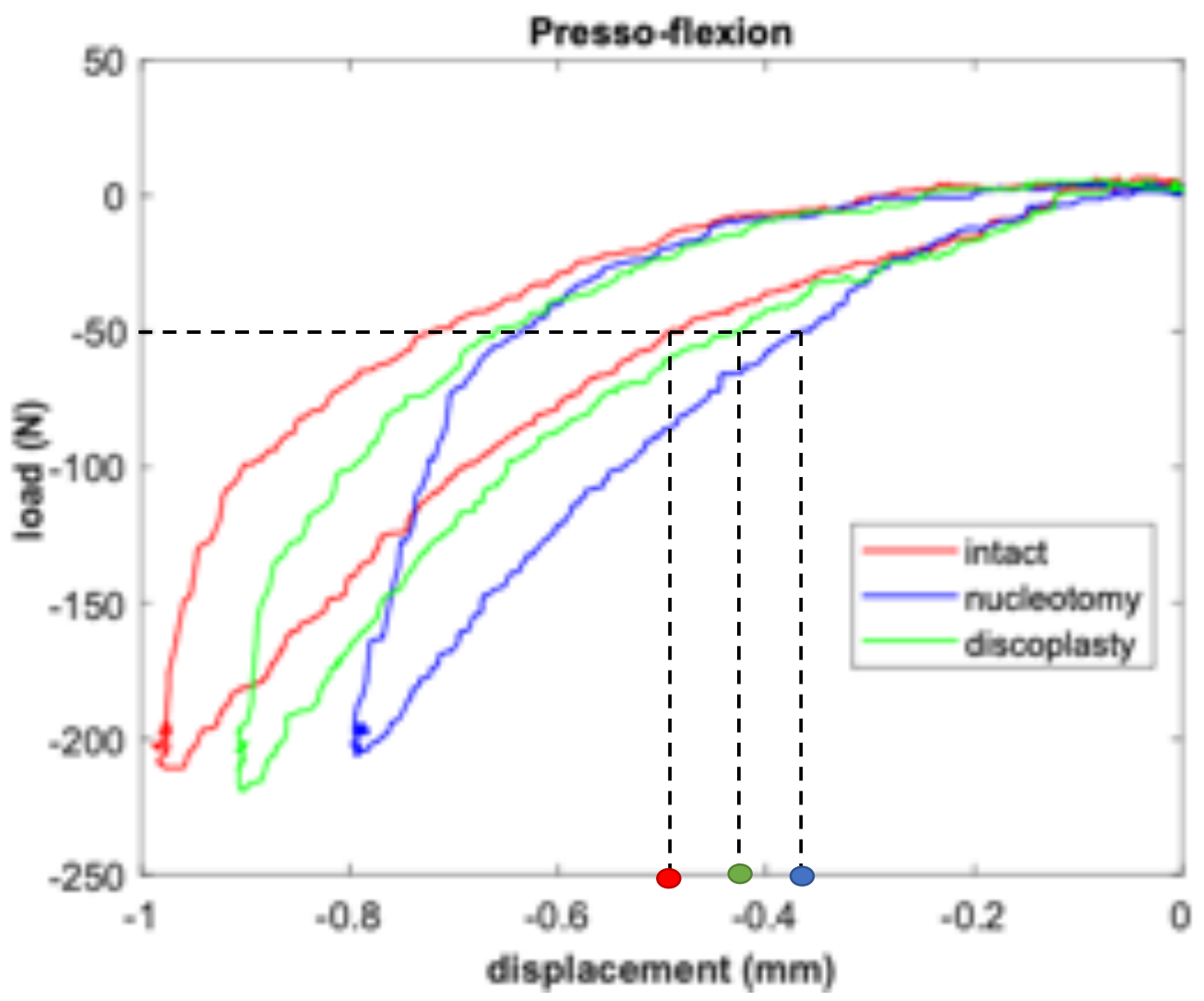

618 Fig. 5 - Load-displacement curve of a representative specimen tested in extension in 619 all disc conditions. 
4.0

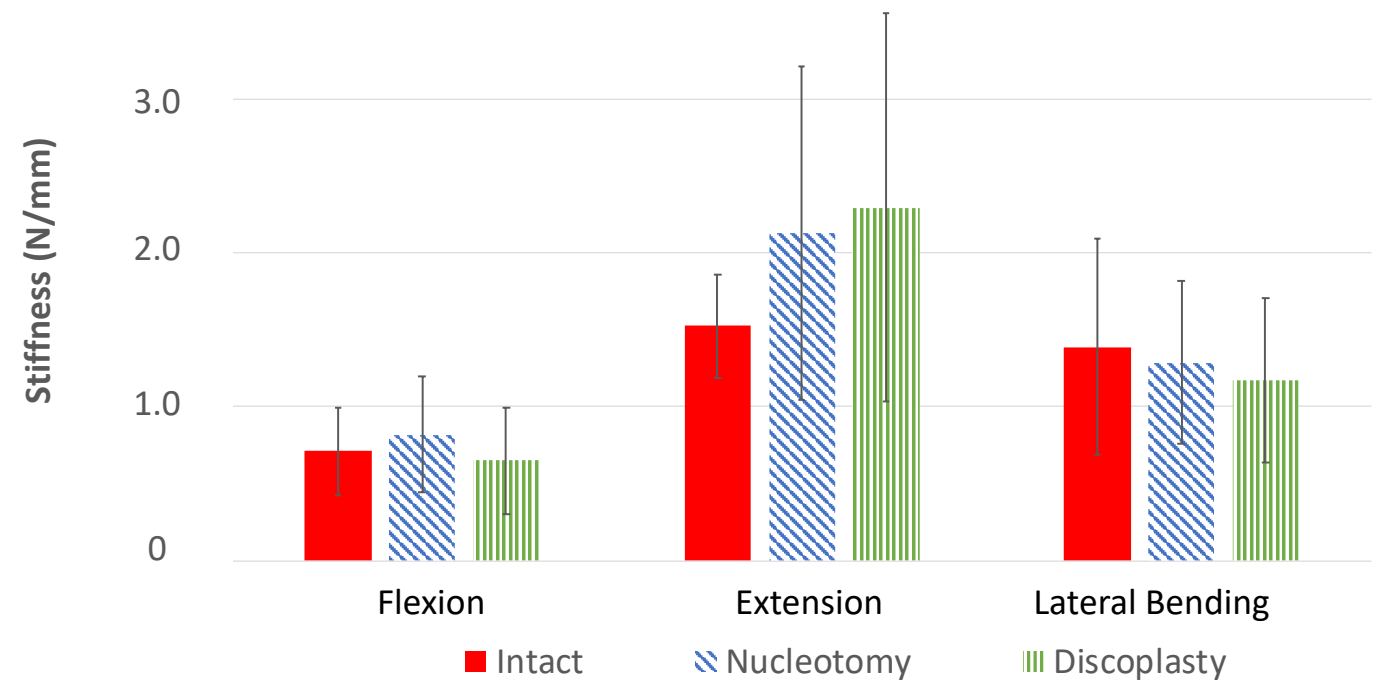

621

622 Fig. 6 - Stiffness results in all conditions for all loading configurations. Average was 623 done over all specimens. Normalized data were not statistically significant ( $p>0.1)$. 


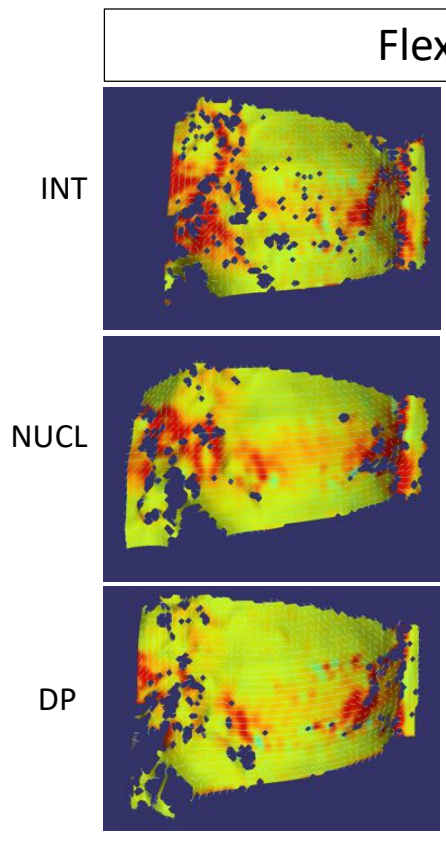

$\varepsilon 1$
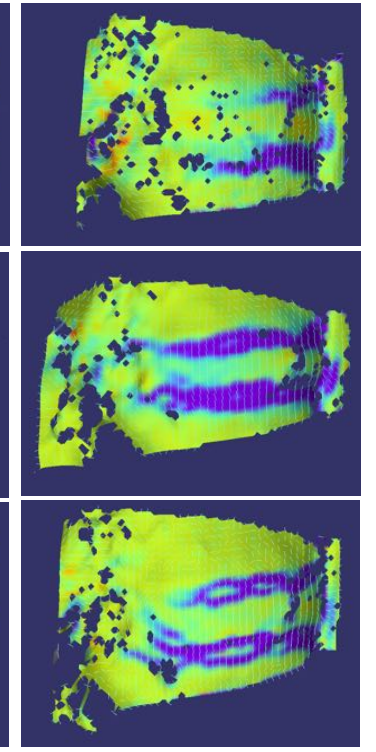

$\varepsilon 2$

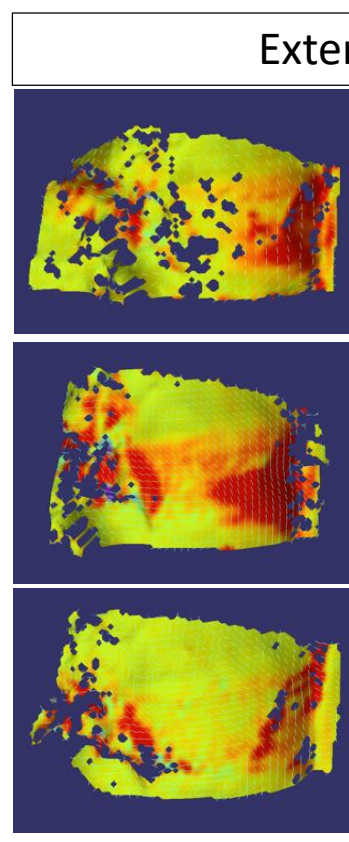

$\varepsilon 1$
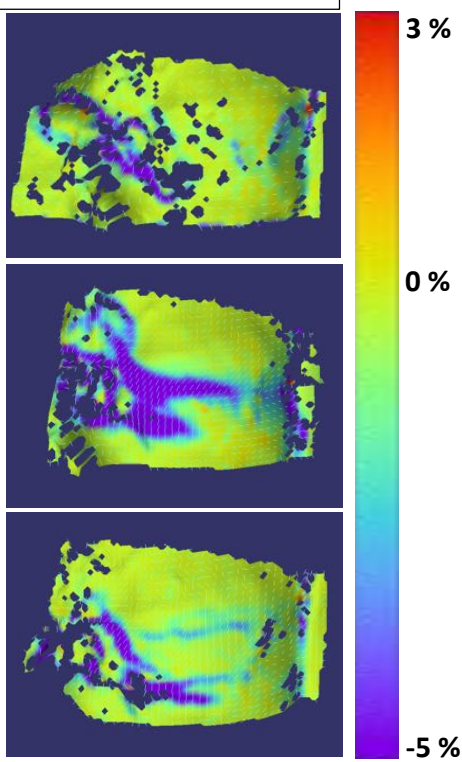

$\varepsilon 2$

626 Fig. 7 - Showed a typical strain distribution over a specimen surface for a flexion (left)

627 and extension (right) bending with first and second principal strains represented for 628 each motion. 


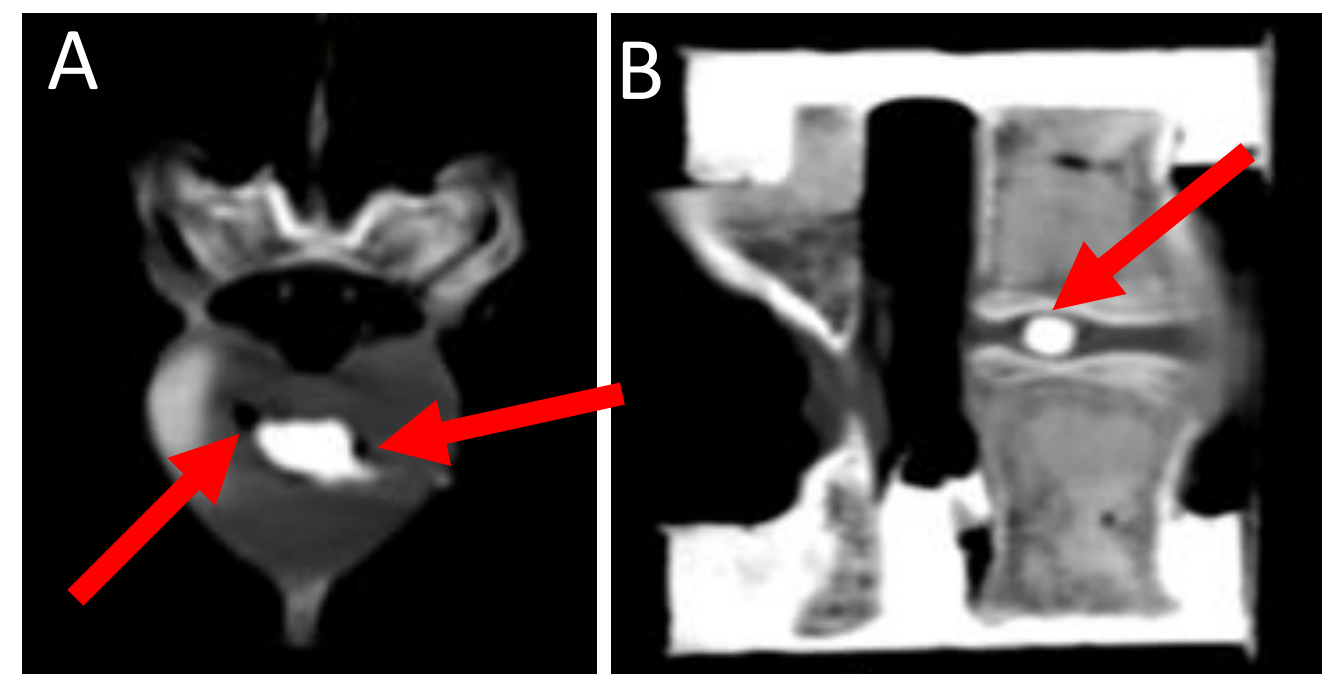

630

631 Fig. 8 -Sub optimal cement distribution. CT scans of porcine specimens in axial (A) 632 and sagittal (B) planes. PMMA did not reach the annulus and the endplates (arrows), 633 leaving vacuum.

634 

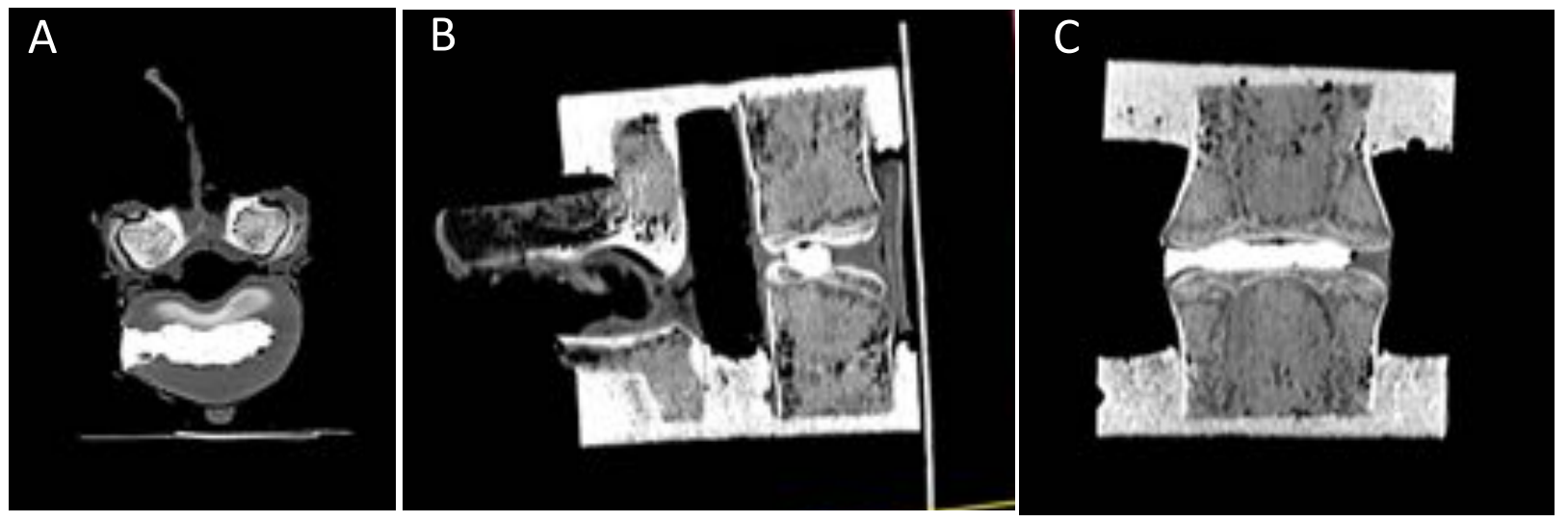

636 Fig. 9 - Ideal distribution of the PMMA in the porcine model. CT scan of the porcine

637 specimen, A (axial plane) the PMMA filled out the empty space after nucleotomy, B

638 (sagittal plane) and C (coronal plane) the PMMA reached the two endplates and adapted 639 to the geometry. 


\section{TABLES}

641 Table 1: Principal strains recorded over the disc surface in Flexion and Extension: The 642 mean and peak (of 10 specimens) are reported for $\mathcal{E} 1$ and for $\mathcal{E} 2$.

643

$\varepsilon 1$

\begin{tabular}{cccc} 
Flexion & \multicolumn{3}{c}{ Extension } \\
\hline Mean $(\%)$ & Peak & Mean & Peak
\end{tabular}

(\%) $\quad(\%)$

\begin{tabular}{lllll}
\hline Intact & $1.3 \pm 0.6$ & $7.5 \pm 2.8$ & $2.2 \pm 1.0$ & $11.7 \pm 6.0$ \\
Nucleotomy & $1.3 \pm 0.7$ & $10.5 \pm 7.1$ & $1.9 \pm 0.6$ & $10.1 \pm 3.9$ \\
Discoplasty & $1.0 \pm 0.5$ & $8.7 \pm 3.5$ & $1.2 \pm 0.7$ & $10.0 \pm 4.1$ \\
\hline
\end{tabular}

\begin{tabular}{lcccc}
\multirow{2}{*}{2} & Flexion & \multicolumn{3}{c}{ Extension } \\
\cline { 2 - 5 } & Mean (\%) & $\begin{array}{c}\text { Peak } \\
(\%)\end{array}$ & $\begin{array}{c}\text { Mean } \\
(\%)\end{array}$ & $\begin{array}{c}\text { Peak } \\
(\%)\end{array}$ \\
\hline Intact & $-2.0 \pm 1.2$ & $-17.2 \pm 6.1$ & $-0.5 \pm 0.4$ & $-8.2 \pm 7.5$ \\
Nucleotomy & $-2.8 \pm 1.6$ & $-18.7 \pm 8.9$ & $-1.7 \pm 1.5$ & $-12.5 \pm 10.4$ \\
Discoplasty & $-1.7 \pm 0.9$ & $-16.5 \pm 7.3$ & $-0.7 \pm 0.8$ & $-13.3 \pm 5.3$ \\
\hline
\end{tabular}


645 Table 2: Surface area and volume of the injected cement after segmentation.

646

\begin{tabular}{cccc}
\hline Specimen & Spine level & $\begin{array}{c}\text { Cement surface } \\
\text { area }\left(\mathbf{m m}^{2}\right)\end{array}$ & $\begin{array}{c}\text { Cement volume } \\
\left(\mathbf{m m}^{3}\right)\end{array}$ \\
\hline$\# 1$ & T13-L1* & 257.8 & 282.8 \\
$\# 2$ & L3-L4 & 465.8 & 476.7 \\
$\# 3$ & T13-L1 ${ }^{*}$ & 211.6 & 143.5 \\
$\# 4$ & L5-L6 & 623.7 & 673.9 \\
$\# 5$ & T13-L1* & 712.3 & 750.3 \\
$\# 6$ & L3-L4 & 552.0 & 608.7 \\
$\# 7$ & L3-L4 & 742.2 & 776.5 \\
$\# 8$ & L3-L4 & 557.6 & 505.4 \\
$\# 9$ & T15-L1 & 592.7 & 685.0 \\
\hline Mean (SD) & - & $524.0(184.2)$ & $544.8(215.7)$
\end{tabular}

$647{ }^{*}$ Porcine spines have a variable number of thoracic vertebrae (between 13 and 15). 